ISSN 0103-5150

Fisioter. Mov., Curitiba, v. 26, n. 3, p. 537-548, jul./set. 2013

Licenciado sob uma Licença Creative Commons

\title{
Comprometimento sensório-motor dos membros inferiores em diabéticos do tipo 2
}

\section{Sensitive-motor alteration of the lower limbs in diabetics type 2}

\author{
Silvia Regina Barrile ${ }^{[\mathrm{a}]}$, Aline Aparecida Ribeiro ${ }^{[\mathrm{b}]}$, Ana Paula Rodrigues da Costa ${ }^{[\mathrm{c}]}$, \\ Ariane Aparecida Viana ${ }^{[\mathrm{d}]}$, Marta Helena Souza De Conti ${ }^{[\mathrm{el}}$, Bruno Martinelli ${ }^{[\mathrm{f}]}$
}

[a] Doutora em Fisiopatologia em Clinica Médica pela Universidade Estadual Paulista (Unesp), câmpus Botucatu, professora do curso de Fisioterapia da Universidade do Sagrado Coração (USC), Bauru, SP - Brasil, e-mail: srbarrile@gmail.com

[b] Especialista em Fisioterapia Dermatofuncional e Saúde da Mulher pela Universidade do Sagrado Coração (USC), fisioterapeuta da Apae de Pederneiras, Pederneiras, SP - Brasil, e-mail: ft.alineribeiro@gmail.com

[c] Graduada em Fisioterapia pela Universidade do Sagrado Coração (USC), Bauru, SP - Brasil, e-mail: aninha_fannie@yahoo.com.br

[d] Especialista em Fisioterapia Hospitalar pela Universidade Estadual Paulista (Unesp), câmpus Botucatu, fisioterapeuta do Hospital Estadual Bauru (HEB), Bauru, SP - Brasil, e-mail: arianeaviana@gmail.com

[e] Doutora em Ginecologia, Obstetrícia e Mastologia pela Universidade Estadual Paulista (Unesp), câmpus Botucatu, professora do curso de Fisioterapia e do mestrado em Saúde Coletiva da Universidade do Sagrado Coração (USC), Bauru, SP - Brasil, e-mail: madeconti@yahoo.com.br

[f] Mestre em Fisiopatologia em Clinica Médica pela Universidade Estadual Paulista (Unesp), câmpus Botucatu, professor do curso de Fisioterapia da Universidade do Sagrado Coração (USC), Bauru, SP - Brasil, e-mail: internetbruno@yahoo.com.br

\section{Resumo}

Introdução: A alta incidência do Diabetes mellitus (DM) na população torna essa patologia alvo de muitas pesquisas. Uma das complicações do DM é a neuropatia periférica crônica caracterizada por atrofia e degeneração axonal e/ou alteração da célula de Schwann. A fisioterapia atua na estimulação da regeneração da fibra nervosa, o que reduz o risco de desenvolvimento do pé diabético. Objetivo: Identificar o comprometimento da sensibilidade nos membros inferiores (MMII) de diabéticos e verificar fatores que contribuíram para o desenvolvimento do pé diabético, como glicemia, hemoglobina glicada (HbA1c), tempo de diagnóstico, pressão arterial e antropometria. Materiais e métodos: Os diabéticos foram avaliados na Associação dos Diabéticos de Bauru, participaram de entrevista e foram submetidos a antropometria, triagem pressórica e teste neurossensorial no Sistema Pontuação Clínica Toronto (SPCT). Resultados: Os 68 pacientes (61,76\% mulheres e 38,23\% homens) tinham 62,93 \pm 9,64 anos, apresentaram pressão sistólica de 132,54 \pm 17,95 mmHg e diastólica 80,39 \pm 11,35 mmHg, índice de massa 
corpórea 30,34 \pm 5,80 kg/m², glicemia 149,20 \pm 60,37mg/dL, HbA1c 7,72 \pm 1,4\%; tempo de diagnóstico de um a 34 anos. Dentre eles, 80,88\% apresentavam sobrepeso; 72,06\%, hipertensão arterial; 42,64\%, dislipidemia; $14,7 \%$, problemas renais; e 17,64\%, vasculares. Pelo SPCT, 97,05\% apresentavam neuropatia, 95,59\% com simetria. Na avaliação, $57,35 \%$ apresentavam alteração sensitiva protetora; $60,29 \%$, sensibilidade térmica; $26,47 \%$, tátil; $17,64 \%$, vibratória; 7,35\%, dolorosa; e 1,47\%, alteração cinestésica. Nos pés, 72,05\% apresentavam pele ressecada; $64,70 \%$, fissuras; 54,41\%, calosidade; e 44,11\%, micose; $50 \%$ referiram dor; 39,7\%, dormência; $41,17 \%$, parestesia; $27,94 \%$, ataxia. Reflexos estavam ausentes ou diminuídos em $14,71 \%$ dos indivíduos; desses, 38,23\% no Aquileu. Observou-se correlação positiva entre o SPCT com a glicemia e a circunferência abdominal e a hemoglobina glicada com a glicemia. Conclusão: Houve grande incidência de neuropatia simétrica; alteração de sensibilidade com presença de sinais clínicos, sintomas, arreflexia e hiporeflexia; e incidência de obesidade e hipertensão associadas. Faz-se necessário o acompanhamento da fisioterapia na avaliação da sensibilidade para prevenir, reduzir sintomas e melhorar a circulação sanguínea em MMII.

Palavras-chave: Neuropatias diabéticas. Fisioterapia. Avaliação. Sensibilidade.

\section{Abstract}

Introduction: The high incidence of diabetes mellitus (DM) in the population becomes this pathology subject of much research. One complication of diabetes is peripheral neuropathy characterized by chronic atrophy and axonal degeneration and/or alteration of the Schwann cell. The physiotherapy works in the stimulation of regeneration of the nerve fiber, which reduces the risk of developing diabetic foot. Objective: To identify the sensitivity disorders in the lower limbs (LL) in diabetics patients and verify factors that contribute to the development the diabetic foot as glicemic level, glycosylated hemoglobin (GH), time of the diagnostic, blood pressure, anthropometry and life habits. Materials and methods: The diabetics patients were valued in Bauru Diabect Association and submitted the interview, anthropometry, blood pressure, by the Toronto Clinical Scoring System avaliation (TCSS - sensorial test, reflexes e symptoms). Results: The 68 enrolled patients, $61.76 \%$ female and $38.23 \%$ male, with $62.93 \pm 9.64$ years-old, showed systolic blood pressure of $132.54 \pm 17.95 \mathrm{mmHg}$ and diastolic blood pressure of $80.39 \pm 11,35 \mathrm{mmHg}$; body mass index, 30,34 $\pm 5,80 \mathrm{~kg} / \mathrm{m} 2$; glicemic level, $149,20 \pm 60,37 \mathrm{mg} / \mathrm{dL}$; GH, 7,72 $\pm 1,4 \%$; time of the diagnostic, one to 34 years; 80,88\% had overweight, 72,06\%, hypertension; 42,64\%, dyslipidemia; 14,7\%, kidney; and 17,64\%, vascular disorders. According to TCSS, 97.05\% showed neuropathy, 95.59\% were symmetrical. Regarding the sensitivity altered, $57.35 \%$ showed in protect; $60.29 \%$, thermal; $26.47 \%$, tactile; $17.64 \%$, vibratory; $7.35 \%$, painful; and $1.47 \%$, kinectic-postural. In the feet, $72.05 \%$ had dry foot skin; $64.70 \%$, fissures; $54.41 \%$, callosity; $44.11 \%$, mycosis; 50\% reported pain; 39,7\%, numbness; 41,17\%, tingling; 27,94\%, sensory ataxia. The reflexes were abolished or decreased in 14.71\%; 38.23\% are localized in Achilles tendon. There was positive correlation between the TCSS with abdominal circumference and blood glucose and glycated hemoglobin with glucose. Conclusions: There was great incidence of the symmetrical neuropathy; sensibility alteration with clinical manifestations, symptons, areflexia and hyporeflexia; obesity and hypertension incidences were associated. It is very necessary the physiotherapy accompaniment on the sensitivity valuation to prevent, reduce the symptons and improve the blood circulation in $L L$.

Keywords: Diabetic neuropathies. Physical therapy. Evaluation. Sensitivity.

\section{Introdução}

Diabetes mellitus (DM) é uma doença que tem como característica principal a falta absoluta ou relativa de insulina no organismo (1), compreende um grupo heterogêneo de causas e manifestações clínicas, ocasionando modificações no metabolismo de proteínas, gorduras, sais minerais e principalmente glicose (2). O DM 2 é mais comum, sendo causado por acentuada redução da sensibilidade dos tecidos-alvo aos efeitos metabólicos da insulina, hormônio produzido pelas células $\beta$ do pâncreas e secretado quando há glicose no sangue, apresentando como função primordial seu transporte para o interior das células (3). Em células específicas como as do tecido nervoso, particularmente células de Schwann, da retina, ilhotas de Langerhans no pâncreas, 
papilas renais e eritrócitos, a proteína responsável pela entrada de glicose é o GLUT 2. Esse transportador não necessita do estímulo da insulina para a entrada de glicose na célula (4).

Na presença de hiperglicemia ocorre alta concentração de glicose dentro das células específicas citadas, gerando alterações que identificam áreas patologicamente afetadas nos diabéticos, com formação de retinopatia, nefropatia e neuropatia diabética (4).

Segundo Cunha et al. (1), a neuropatia é uma das piores complicações crônicas do DM, sendo um distúrbio no nervo periférico caracterizado por atrofia e degeneração axonal e/ou por alterações nas células de Schwann, provocando desmielinização em segmentos do axônio, tendo como consequência diminuição da velocidade de condução ou interrupção da transmissão do impulso nervoso.

De acordo com Rull et al. (5), numerosos estudos histopatológicos realizados em indivíduos diabéticos têm demonstrado a existência de lesões nos sistemas nervoso periférico e autônomo. As lesões têm sido identificadas nas células de Schwann, células perineurais, nos axônios e nos elementos vasculares endoneurais. Todas elas podem participar no desenvolvimento da neuropatia diabética, especialmente em sua forma clínica mais comum, a polineuropatia distal e simétrica.

Segundo Schmid et al. (6), a neuropatia sensitivo-motora tem início insidioso, os sinais e sintomas variam de acordo com o tipo de fibra envolvida. Segundo Sacco et al. (7), a neuropatia diabética gera transtornos tróficos da pele e osteoarticulares do pé, como atrofia muscular e diminuição da amplitude de movimento, o que resulta em risco de ulceração plantar.

A detecção precoce e o tratamento oportuno das manifestações clínicas podem evitar metade das amputações em indivíduos portadores de DM, estudos mostraram que programas educacionais abrangentes podem prevenir metade das ocorrências de amputação (8).

A maior parte dos custos com o DM relaciona-se às suas complicações, sendo que estas podem ser reduzidas e evitadas pela avaliação das extremidades inferiores dos diabéticos de forma minuciosa e com frequência regular (9).

Úlceras superficiais ou lesões pré-ulcerativas nos pés de indivíduos diabéticos com diminuição de sensibilidade causada por neuropatia diabética constituem 85\% dos casos graves de internações hospitalares (10).

Conforme comprovado por Bril e Perkins (11), o Sistema de Pontuação Clínica de Toronto (SPCT) para a neuropatia diabética mostrou-se um método simples para demonstrar a presença e a severidade da neuropatia diabética, obtendo-se diagnóstico mais fidedigno.

A fisioterapia atua na avaliação do risco de desenvolvimento da neuropatia diabética e na estimulação da regeneração da fibra nervosa.

O objetivo deste estudo foi classificar a sensibilidade dos membros inferiores (MMII) em indivíduos diabéticos e correlacionar com fatores que contribuem para o desenvolvimento do pé diabético (glicemia de jejum, hemoglobina glicada (HbA1c), tempo de diagnóstico do DM, pressão arterial, antropometria e hábitos de vida).

\section{Materiais e métodos}

Neste tópico serão apresentadas as estratégias metodológicas envolvendo as técnicas e materiais utilizados neste estudo a fim de alcançar os objetivos propostos.

\section{População de estudo}

Foi realizado um estudo de coorte utilizando amostragem intencional (12) com convite dirigido aos indivíduos frequentadores da Associação dos Diabéticos de Bauru (ADB) com presença ou ausência de sinais e sintomas de polineuropatia diabética.

Foram incluídos nesse estudo indivíduos portadores de DM 2 com diagnóstico médico, cadastrados na Associação dos Diabéticos de Bauru, de ambos os sexos, independentemente da idade, e que aceitaram participar do presente estudo, com a assinatura do Termo de Consentimento Livre e Esclarecido.

Os indivíduos cadastrados na Associação dos Diabéticos de Bauru (ADB) foram convidados por meio de telefonemas e durante as reuniões mensais realizadas no terceiro sábado de cada mês.

O trabalho foi aprovado pelo Comitê de Ética em Pesquisa na Pró-Reitoria de Pesquisa e Pós-Graduação da Universidade Sagrado Coração (USC), sob o protocolo n. 138/07.

Aos que concordaram em participar do presente estudo aplicou-se um questionário elaborado pelos autores, para identificar comorbidades como hipertensão arterial, dislipidemia, obesidade e hábitos dos indivíduos como etilismo e tabagismo. No questionário 
também foram investigados o tempo de diagnóstico de DM, antecedentes familiares, ocupação e cuidados para o controle do DM, como dieta e prática de exercícios físicos. Em seguida, os indivíduos foram submetidos a avaliação físico-funcional, realizada por fisioterapeutas.

\section{Avaliação físico-funcional}

A avaliação físico-funcional foi composta pela mensuração das dimensões corpóreas, níveis pressóricos (pressão arterial sistêmica), testes sensoriais profundos e periféricos além dos níveis glicêmicos.

\section{Antropometria}

A avaliação antropométrica consistiu de avaliação do peso (kg), obtido por meio de balança antropométrica digital (FILIZOLA, São Paulo, Brasil), calibrada a cada medida, com precisão de $0,1 \mathrm{~kg}$. Para a mensuração os indivíduos permaneceram descalços, com o mínimo de roupa. Para altura (m) utilizou-se o estadiômetro presente na balança, com precisão de $0,5 \mathrm{~cm}$, com os indivíduos descalços, e pés unidos, com calcanhares e costas apoiados na parede, a cabeça ereta e em plano horizontal, com esquadro móvel pousado suavemente sobre ela. O índice de massa corpórea (IMC) foi calculado a partir das medidas do peso corporal $(\mathrm{kg})$, e estatura (m), pela fórmula: $\mathrm{IMC}=$ peso $/$ estatura $^{2}\left(\mathrm{~kg} / \mathrm{m}^{2}\right)(13)$.

A circunferência abdominal (CA) foi verificada com auxílio de fita métrica, de material inextensível e precisão de $0,5 \mathrm{~cm}$, sendo devidamente posicionada com base em referenciais preestabelecidos, mais precisamente no meio da distância entre a crista ilíaca e o rebordo costal inferior (14).

\section{Triagem pressórica}

Para verificação dos níveis de pressão arterial (PA), os indivíduos permaneceram sentados em uma cadeira com as costas apoiadas, mantendo as pernas descruzadas e braço na altura do coração. Os avaliadores se certificaram sobre a condição do indivíduo de não estar com a bexiga cheia, e da não realização de prática de exercícios físicos, ingestão de bebidas alcoólicas, café, alimentos ou fumo até 30 minutos antes da aferição. Ao indivíduo foi solicitado descansar por 5 a 10 minutos. Utilizou-se manguito de tamanho adequado (bolsa de borracha com largura $=40 \%$ e comprimento $=80 \%$ da circunferência do braço). Estimou-se o nível da pressão sistólica pela palpação do pulso radial; o seu reaparecimento corresponde à pressão arterial sistólica (método palpatório). A campânula do estetoscópio foi posicionada sobre a artéria braquial. 0 manguito foi inflado rapidamente até ultrapassar 20 a 30 mmHg do nível estimado da pressão sistólica, sendo desinflado lentamente. Determinou-se sistólica no aparecimento dos sons de Korotkoff e a diastólica no desaparecimento dos mesmos (15).

As aferições de PA foram realizadas com manômetro aneroide, da marca Lane Instruments, recentemente calibrado para garantir a precisão da mensuração. Foram registradas a pressão arterial sistólica (PAS) e a diastólica (PAD).

\section{Sistema de Pontuação Clínica de Toronto}

Este instrumento é validado e reflete a presença e severidade da polineuropatia periférica diabética sensório-motora. Fez parte desse protocolo o teste sensorial, os testes de reflexos e sintomas referidos (11).

Os indivíduos foram questionados sobre as características da neuropatia diabética como presença ou ausência de dor em queimação e/ou em pontada, dormência, parestesia, fraqueza nos pés, ataxia e presença ou ausência de sintomas similares nos membros superiores.

O teste sensorial foi realizado no hálux e descrito como normal ou anormal. A sensibilidade dolorosa foi avaliada utilizando alfinetes; a térmica foi testada por meio de tubo de ensaio com água fria e quente $\left(42^{\circ} \mathrm{C}\right)$ e termômetro para aferição da temperatura da água com escala graduada de 35 a $42{ }^{\circ} \mathrm{C}$; a sensibilidade tátil foi avaliada utilizando-se algodão; a vibratória foi testada com o diapasão de $128 \mathrm{~Hz}$; e a cinestésica, a partir da posição do membro (16).

0 teste para reflexo patelar foi realizado com os indivíduos sentados com as pernas pendentes e joelhos semifletidos e apoiados sobre a mão do examinador, percutiu-se o tendão rotuliano, obtendo-se a extensão da perna pela contração do quadríceps femoral. 0 teste para reflexo aquileu, realizado com o indivíduo sentado com as pernas pendentes, foi obtido quando, percutido o tendão de Aquiles, observou-se contração do tríceps sural com extensão do pé sobre a perna (17). 
Os resultados do SPCT oscilam de um mínimo de 0 (não neuropatia) para um máximo de 19 pontos para o membro avaliado. Seis pontos são obtidos para sintomas; oito, para reflexos de membro inferior; e cinco para o exame sensorial aplicado nos dedos do pé (11).

Testes de sensibilidade (monofilamentos)

Também foram realizados outros testes de sensibilidade, com o Monofilamento de Semmes-Weinstein de 10 gramas, o qual avalia a sensação cutânea e é padronizado para identificar a perda da sensação protetora do pé. Ele representa um logaritmo $(5,07)$ de dez vezes a força (em miligramas) para fazê-lo curvar. Seu número de aplicações varia de 1 a 14 pontos, sendo a face plantar do hálux e das cabeças dos metacarpos as mais referendadas (16).

\section{Exames bioquímicos}

Foi realizada a coleta de sangue mediante a punção da veia antecubital, com agulha e seringas descartáveis. Após a separação do soro ou plasma, esse material foi encaminhado para realização de análise bioquímica. A glicose plasmática foi dosada pelo Método Enzimático Automático, e a hemoglobina glicada (HbA1c) foi aferida pelo método Imunoturbidimetria, utilizando-se o sangue total (18).

Ao final da avaliação, solicitou-se aos indivíduos seus exames bioquímicos mais recentes (até três meses). Aos que não dispunham, exames foram prescritos pelo médico da ADB.

\section{Análise estatística}

Os resultados foram apresentados por meio de estatística descritiva envolvendo distribuição de frequências absoluta e relativa e do Coeficiente de correlação de Spearman (12).

\section{Resultados}

A população do estudo constituiu-se de 68 indivíduos com diagnóstico médico de DM 2, cadastrados na Associação dos Diabéticos de Bauru, sendo $61,76 \%$ mulheres e $38,23 \%$ homens, com média de idade de $62,93 \pm 9,64$ anos. Observou-se predomínio da etnia branca $(69,11 \%)$, seguida da negra $(25 \%)$ e asiática (5,88\%). 0 tempo de diagnóstico da doença variou de 1 a 34 anos $(10,74 \pm 7,52)$.

Quanto aos hábitos de vida, percebeu-se que 33,82\% eram tabagistas, $29,41 \%$ ex-tabagistas e 36,76\% nunca fizeram uso de tabaco. Tinham o hábito de ingerir bebidas alcoólicas com frequência $14,70 \%$ dos indivíduos. Em relação aos cuidados físicos, constatou-se que $51,47 \%$ dos participantes avaliados praticavam exercícios físicos com média de 1,75 $\pm 1,91$ vezes por semana e 83,82\% relataram adesão à dieta hipoglicêmica.

Observou-se que $80,88 \%$ dos indivíduos estavam acima do peso ideal. Notou-se presença de hipertensão arterial sistêmica em $72,05 \%$ dos indivíduos, seguida de dislipidemia em $42,64 \%$, problemas vasculares em 17,64\% e renais em 14,7\% (Gráfico 1).

Quanto à presença de diabéticos na família verificou-se que $33,82 \%$ referiram antecedentes familiares, sendo que 30,88 deram respostas negativas e $35,29 \%$ não souberam responder (Gráfico 2).

Na análise da glicemia observou-se média de 149,20 $\pm 60,37 \mathrm{mg} / \mathrm{dL}$, e hemoglobina glicada de $7,72 \pm 1,4 \%$, dados que definem o controle glicêmico dos indivíduos estudados. Observou-se também correlação positiva entre essas duas variáveis ( $\mathrm{p}=0,000026)$.

A pressão arterial sistólica foi em média 132,54 \pm $17,95 \mathrm{mmHg}$ e diastólica $80,39 \pm 11,35 \mathrm{mmHg}$. 0 peso dos indivíduos na avaliação antropométrica foi em média $77,86 \pm 16,16 \mathrm{~kg}$ e a altura $1,6 \pm 0,08 \mathrm{~m}$. De acordo com a caracterização da amostra estudada, constatou-se que a média do IMC foi de 30,34 \pm 5,8 $\mathrm{kg} / \mathrm{m}^{2}$, sendo possível classificar os indivíduos como eutrofia $(19,11 \%)$, sobrepeso $(26,47 \%)$, obesidade grau I $(33,82 \%)$, obesidade grau II $(17,64 \%)$ e obesidade grau III $(2,94 \%)$.

A medida da CA foi em média 101,32 $\pm 16,98$ cm. Dos 26 homens avaliados, 57,69\% apresentaram CA $\geq 102 \mathrm{~cm}$. Das 42 mulheres avaliadas, $85,71 \%$ apresentaram $C A \geq 88 \mathrm{~cm}$, pontos de corte recomendados pela Adult Treatment Panel III $(19,20)$. Foi possível também apurar que $64,70 \%$ dos indivíduos avaliados apresentavam duas ou mais doenças associadas, sendo, portanto, portadores de Síndrome Metabólica. A circunferência abdominal correlacionou-se significativamente com a pontuação do SPCT de membro inferior esquerdo $(\mathrm{p}=0,009427)$

De acordo com o SPCT, 97,05\% dos indivíduos estudados apresentavam neuropatia diabética variando 


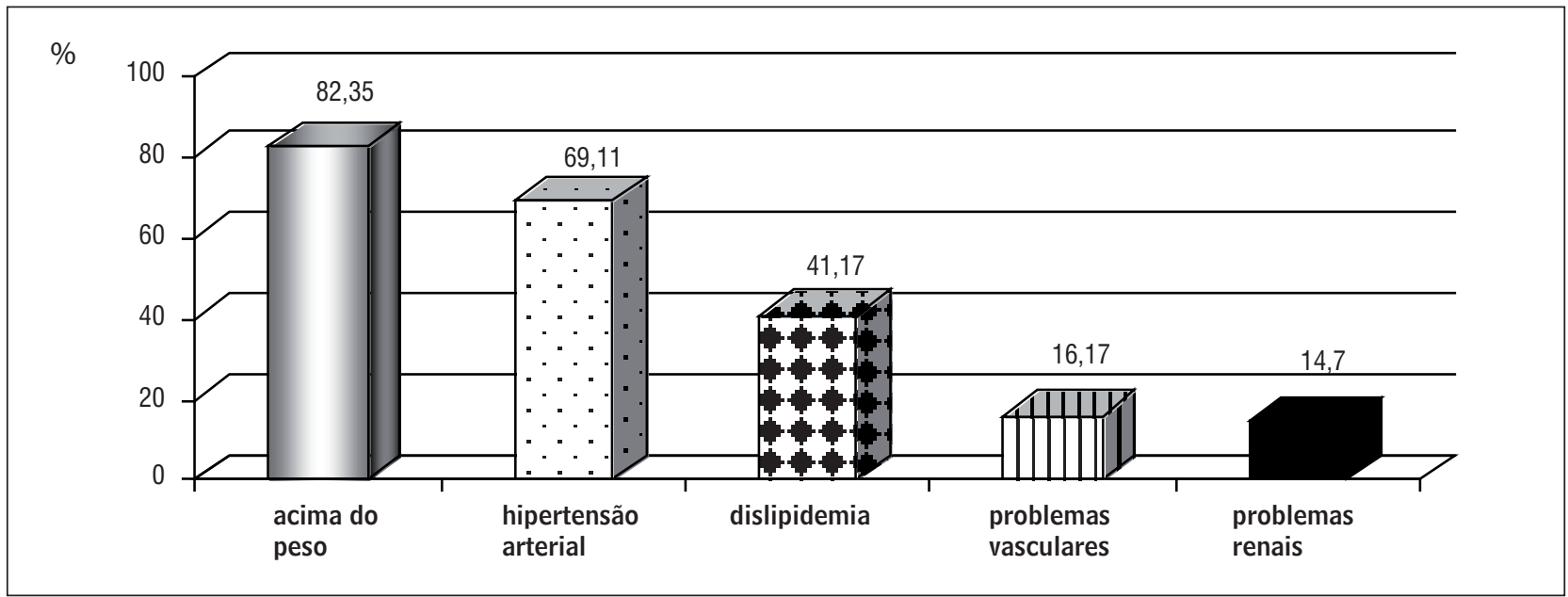

Gráfico 1 - Frequência relativa das comorbidades apresentadas em antecedentes pessoais pela amostra estudada Fonte: Dados da pesquisa.

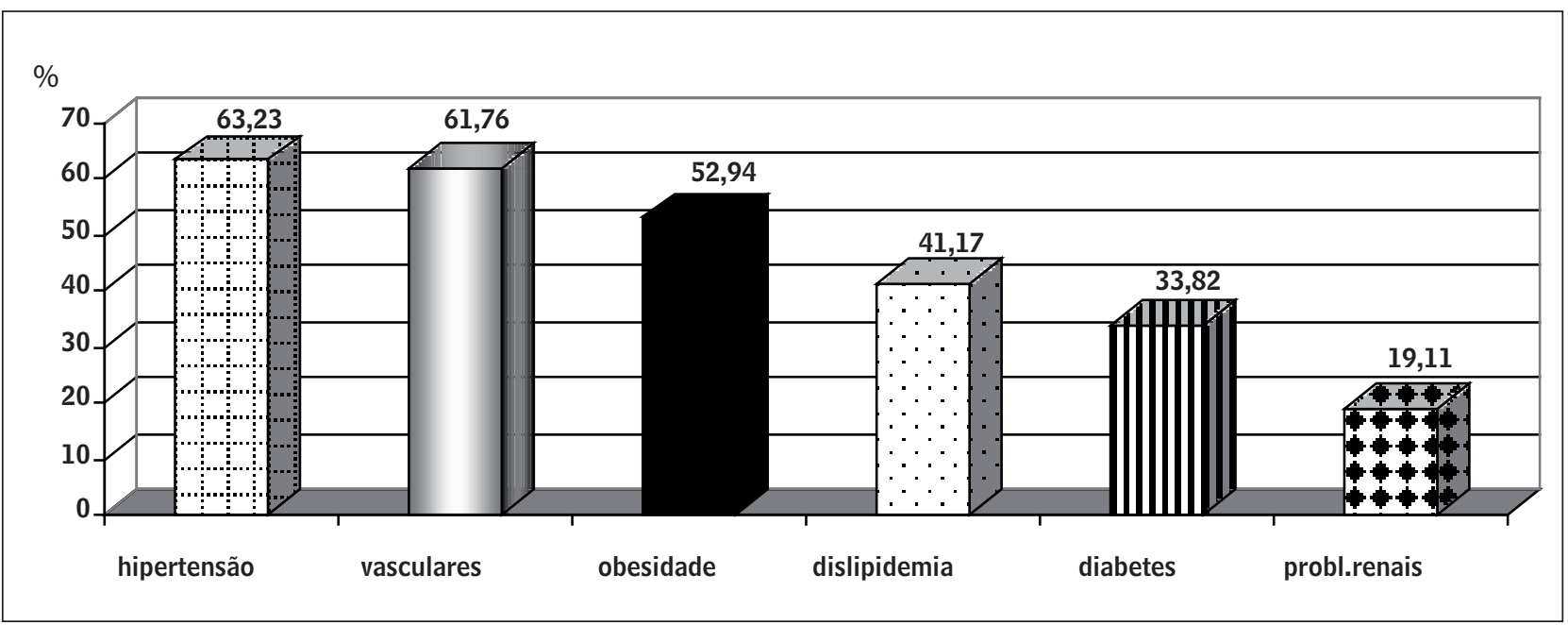

Gráfico 2 - Fatores de risco de antecedentes familiares referidos pelos indivíduos do estudo Fonte: Dados da pesquisa.

de 1 a 12 pontos; destes, 95,58\% apresentavam neuropatia de forma simétrica.

Na análise com emprego do SPCT, 2,94\% apresentaram pontuação igual a 0 , o que significa ausência de neuropatia, $61,76 \%$ apresentaram de 1 a $5,25 \%$ apresentaram de 6 a 8, e finalmente 10,29\% da população estudada apresentou pontuação de 9 a 12 . Observou-se correlação positiva entre a pontuação do SPCT tanto de membro inferior direito $(p=0,031601)$ quanto de membro inferior esquerdo $(p=0,043208)$ com a glicemia.

Na apresentação de sintomas 50\% dos indivíduos referiram dor tanto em membro inferior direito (MID) como em esquerdo (MIE), sendo que dos acometidos 76,47\% apresentavam dor de forma simétrica, 39,71\% relataram dormência em MID e 35,29\% em MIE; 75,86\% dos casos apresentavam simetria.

A presença de parestesia foi relatada em $41,17 \%$ dos indivíduos em MID e 35,29\% em MIE, e 5,88\% dos estudados referiram parestesia em somente um dos membros, sendo o MID o mais afetado em todos os casos.

Da amostra estudada 35,29\% apresentaram fraqueza em MID e MIE, sendo que $8 \%$ dos acometidos apresentavam assimetria.

A ataxia em membro inferior estava presente em $27,94 \%$ em MID e 25\% em MIE; $80 \%$ dos acometidos apresentaram simetria (Gráfico 3). 


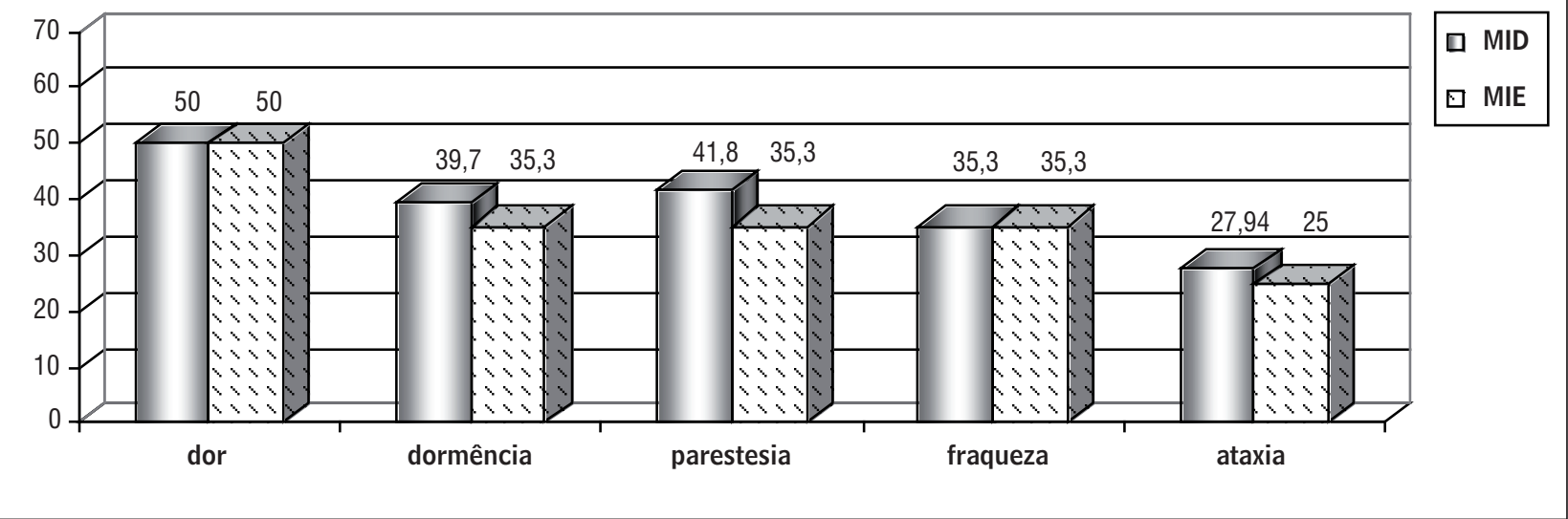

Gráfico 3 - Incidência de sintomas em membro inferior direito (MID) e esquerdo (MIE)

Fonte: Dados da pesquisa.

Entre os indivíduos estudados, 32,82\% referiram sintomas em membro superior direito e $35,29 \%$, em esquerdo; e 67,86\% apresentaram os sintomas em um só dos membros (assimetria), com predomino em membro superior esquerdo $(17,86 \%)$.

O reflexo patelar estava ausente em $13,24 \%$ dos estudados em MID e 11,76\% em MIE, diminuídos 16,18\% em MID e 20,59\% em MIE e 70,59\% e 67,65\% apresentavam normorreflexia em MID e MIE, respectivamente. Da população analisada, $68 \%$ apresentaram acometimento simétrico; e nos casos de assimetria, o predomínio foi em MIE com $20 \%$ dos casos com alteração.

Dos indivíduos estudados, 14,71\% apresentaram arreflexia do Aquileu em MID e 10,29\% em MIE, 29,41\% dos casos apresentaram hiporreflexia em MID e $38,23 \%$ em MIE, 55,88\% e 51,47\% apresentaram normoreflexia em MID e MIE, respectivamente. Nos casos de assimetria, o predomínio de acometimento foi em MIE com 14,29\% dos casos (Gráfico 4).

No teste sensorial, 7,35\% dos indivíduos não apresentaram sensibilidade dolorosa em MID e 5,88\% em MIE, com acometimento simétrico em $80 \%$ dos casos. Houve alteração na sensibilidade térmica de $58,82 \%$ dos estudados, que apresentavam alteração em MID e 60,29\% em MIE, sendo que o acometimento de $76,09 \%$ foi simétrico e $23,91 \%$ assimétrico.

Evidenciou-se comprometimento da sensibilidade tátil em MID em 26,47\% dos indivíduos, e em MIE, em 19,11\%; 72,23\% dos casos eram simétricos. Dos

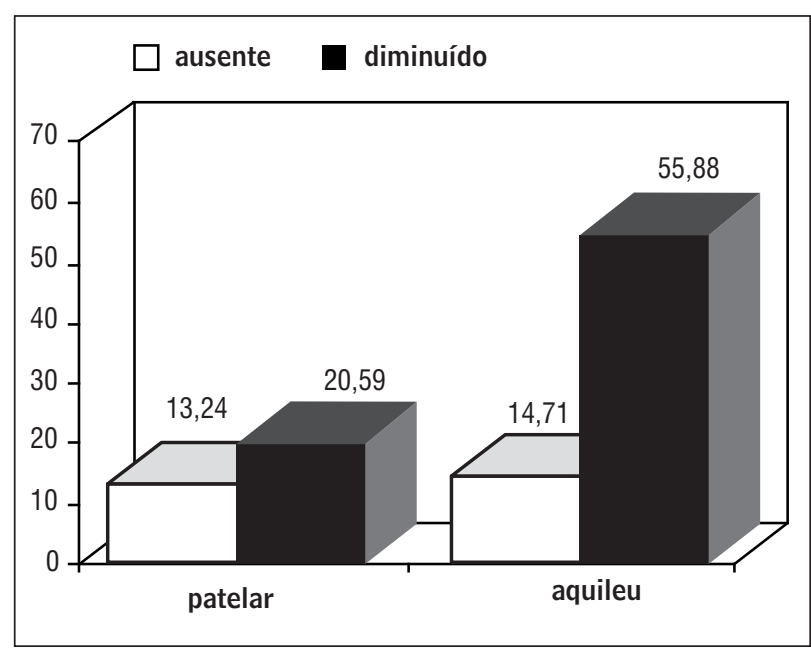

Gráfico 4 - Incidência de reflexos ausentes ou diminuídos na avaliação dos reflexos patelar e aquileu

Fonte: Dados da pesquisa.

estudados, 17,64\% apresentavam alteração da sensibilidade vibratória em MID e 14,70\% em MIE; e apenas 1,47\% apresentavam alteração cinestésica em MID e MIE.

$\mathrm{Na}$ avaliação da sensibilidade com monofilamentos (sensibilidade protetora), 52,94\% apresentavam alteração em MID, e 57,35\% em MIE; observou-se também que $78,57 \%$ da população com alteração apresentava acometimento simétrico (Gráfico 5).

Em relação à inspeção dos pés, 72,05\% apresentavam pele ressecada; $64,70 \%$, fissuras; $54,41 \%$, calosidade; e $44,11 \%$, micose. 


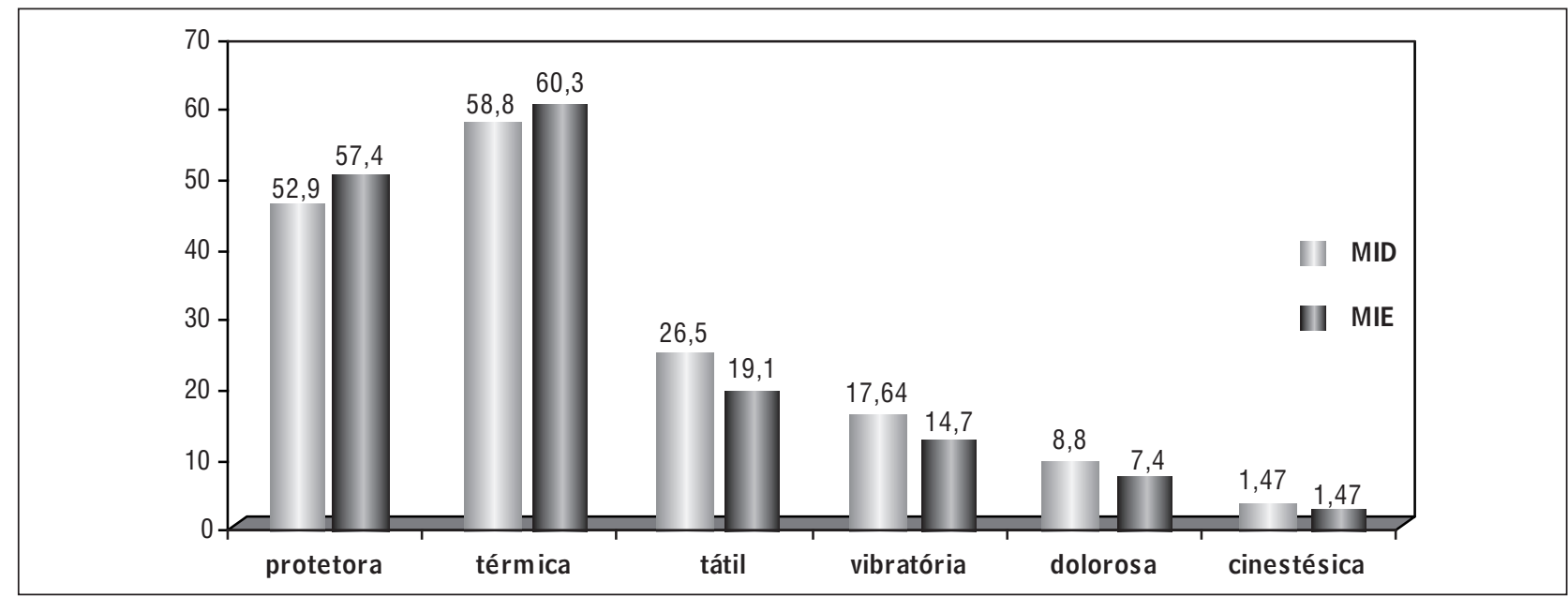

Gráfico 5 - Incidência/distribuição absoluta da preservação da sensibilidade protetora (monofilamento), térmica, tátil, vibratória, dolorosa e cinestésica nos diabéticos

Fonte: Dados da pesquisa.

\section{Discussão}

Diabetes mellitus 2 é uma doença de caráter crônico que aumenta sua incidência proporcionalmente com a idade. No presente estudo obteve-se na população estudada média de idade de 62,93 \pm 9,64 anos, sendo considerada população idosa. Os dados confirmam o predomínio de uma população idosa no Brasil, na faixa de 60 a 69 anos. Esse é o grupo etário populacional de maior crescimento nas últimas décadas no país (20). A predominância do sexo feminino $(61,76 \%)$ confirma a maior expectativa de vida desse grupo, isso pode ser explicado pelo fato de as mulheres se preocuparem mais com o autocuidado do que os homens.

A duração da doença constitui importante fator de risco para o agravo em membros inferiores (9). Neste estudo, perccebeu-se que o tempo de diagnóstico variou de um a 34 anos $(10,74 \pm 7,52)$. Dos indivíduos avaliados, 58,82\% tinham 10 anos ou mais de diagnóstico da doença. Esse fator pode ter colaborado para a grande incidência de neuropatia periférica encontrada na população estudada (21).

A severidade da neuropatia periférica, frequente em diabéticos, se relaciona com outros fatores além do controle glicêmico e duração da doença. Os hábitos de vida dos indivíduos podem agravar a progressão do processo patológico ao colaborar com o decréscimo do fornecimento de oxigênio aos tecidos $(11,22)$.

Considerando que $33,82 \%$ dos indivíduos avaliados neste estudo eram tabagistas e $29,41 \%$ eram ex-tabagistas, deve-se levar em conta que esse fator pode estar relacionado com a diminuição de sensibilidade em membros inferiores. A nicotina desencadeia respostas adrenérgicas que aumentam os valores de glicose, suprimindo a produção de insulina (23). 0 vasoespasmo provocado pela nicotina aumenta a possibilidade de formação de trombos e pode causar insuficiência vascular periférica (24). Dados da literatura identificaram que fumantes estão mais expostos ao risco de amputação de membros inferiores (25).

Quanto ao consumo de bebidas alcoólicas, 14,70\% dos indivíduos relataram consumo frequente. A ingestão de álcool gera um nível intermediário de trocas metabólicas que favorecem o aparecimento de disfunções do transporte axonal que decursa em neuropatia axonal periférica (26). Porém, estudos realizados por Gamba et al. (24) e Laurindo et al. (9) não verificaram correlação entre o hábito de ingerir bebida alcoólica e a ocorrência de complicações em membros inferiores.

0 exercício físico melhora a tolerância à glicose $\mathrm{e}$ a sensibilidade à insulina (7) além de contribuir para melhor circulação nos nervos periféricos, facilitando a perfusão neural e reduzindo a hipóxia endoneural, o que permite melhora na condução nervosa (26). Estudo experimental realizado por Luciano (27) evidenciou considerável redução na hiperglicemia de ratos diabéticos treinados. No presente estudo, $51,47 \%$ dos indivíduos avaliados relataram praticar exercícios físicos com média de $1,75 \pm 1,91$ vezes por semana. Pode-se inferir que apesar dos conhecidos 
benefícios do exercício físico nesses indivíduos, o relato da prática do mesmo não evitou a incidência de neuropatia encontrada no grupo.

Na população diabética estudada, $83,82 \%$ relataram adesão à dieta hipoglicêmica. Estudo realizado por Klack et al. (28) comprovou a eficácia da intervenção nutricional no tratamento de indivíduos diabéticos com redução do IMC, dos níveis de glicose plasmática e da hemoglobina glicada. As autoras destacam a importância de a dieta ser elaborada individualmente para que o controle metabólico adequado seja atingido. Em nosso estudo, a adesão à dieta parece não ter influenciado a redução de peso, pois verificamos que apenas $19,11 \%$ dos indivíduos eram eutróficos (IMC entre 18,5 a $24,9 \mathrm{~kg} / \mathrm{m}^{2}$ ). Sabe-se que a obesidade aumenta a resistência à insulina e a intolerância à glicose, exacerbando as anormalidades metabólicas no diabetes mellitus 2 , aumentando o risco cardiovascular (29).

Outro fator considerado relevante neste estudo foi a presença de Síndrome Metabólica (SM) em 64,70\% dos indivíduos avaliados. Entre os fatores de risco para complicações crônicas do diabetes a presença de SM é significante para as doenças cardiovasculares e também para microangiopatias (30).

A hipertensão arterial foi a comorbidade mais encontrada nos indivíduos avaliados (72,05\%). A hipertensão arterial também aumenta as chances de desenvolvimento de complicações macro e microvasculares e sua associação com as dislipidemias corrobora com o desenvolvimento de doenças macrovasculares, importantes na etiologia das úlceras em extremidades inferiores (9).

A maioria dos indivíduos hipertensos deste estudo realizavam tratamento com anti-hipertensivos, o que explica a média da pressão arterial sistólica de 132,54 \pm 17,95 mmHg e da diastólica 80,39 \pm 11,35 mmHg na triagem pressórica.

No presente trabalho, observou-se a incidência de calosidade e fissuras nos pés dos indivíduos avaliados, sinal determinante de comprometimento autonômico em que há a redução ou supressão da produção de suor nos pés resultando em rachaduras e fissuras (31). A presença de micose e ressecamento nos pés mostra que não é somente um acometimento decorrente de descuido ou desatenção do indivíduo, mas de alterações que os indivíduos diabéticos não controlados podem apresentar.

A neuropatia autonômica cardiovascular ocorre quando há lesão das fibras autonômicas periféricas (simpático e parassimpático) relacionadas ao sistema cardiovascular, resultando em distúrbios na sua regulação neuro-humoral. A maioria dos indivíduos com polineuropatia diabética apresentam algum grau de disfunção autonômica. Aqueles indivíduos com predominância dos sinais e sintomas autonômicos são considerados portadores de neuropatia autonômica diabética. Embora esta possa afetar qualquer sistema orgânico, geralmente se inicia pelos sistemas neurovascular cutâneo (microcirculação dos pés) e cardiovascular (32). A neuropatia autonômica contribui para um mau prognóstico da doença cardíaca, ocorrendo geralmente manifestações relacionadas a lesões do sistema cardiovascular, diminuição da qualidade de vida, e mesmo morte súbita por arritmia cardíaca (33).

Observou-se no grupo estudado incidência de neuropatia diabética, sendo que $97 \%$ dos avaliados tinham algum sinal ou sintoma da patologia. 0 mais preocupante é que a maioria desconhecia a presença desse problema. A neuropatia pode ser identificada logo no inicio, e a prevenção deve ser reforçada, assim como os aspectos clínicos da patologia devem ser estabelecidos a fim de evitar complicações que acarretam altos custos em termos financeiros e diminuição na qualidade de vida dos indivíduos. Em estudo semelhante, Lira et al. (34) teve como objetivo principal determinar a prevalência da polineuropatia em adultos no momento do diagnóstico do diabetes mellitus 2; os autores observaram que dos 113 indivíduos estudados, 29 apresentavam polineuropatia, um dado bastante alarmante, visto que mesmo sem ter o conhecimento da patologia o indivíduo já apresentava alterações crônicas da doença.

Foi observada grande incidência de alteração na sensibilidade protetora dos pés, sendo que $52,94 \%$ em MID e 57,35\% em MIE dos indivíduos não relataram sentir a pressão do monofilamento de 10 gramas nos pontos testados, isso se refere já a comprometimento de fibras sensitivas e autonômicas.

As fibras autonômicas estão comprometidas nos vários subtipos clínicos de neuropatias diabéticas (35). No tipo mais comum (polineuropatia clássica: simétrica, distal e predominantemente sensitiva), há forte correlação entre a lesão progressiva das fibras somáticas e autonômicas, isto é, hoje se sabe que 50\% dos diabéticos portadores de polineuropatia têm neuropatia autonômica cardiovascular assintomática, e $100 \%$ dos portadores de neuropatia autonômica cardiovascular sintomática apresentam polineuropatia clássica. 
Em relação aos sintomas, os de maior frequência foram os de dor e dormência, fato também observado por Sacco et al. (7) num estudo em que $80 \%$ da população estudada apresentava a mesma sintomatologia. A diminuição do aporte sanguíneo nas extremidades de MMII contribui para uma circulação precária nos nervos periféricos, ocorrendo hipóxia endoneural e diminuição da condução nervosa, com piora dos sintomas.

Quanto à avaliação dos reflexos, observou-se grande número de indivíduos com hipo e arreflexia do patelar e aquileu, tendo predomínio a perda do reflexo aquileu. $\mathrm{O}$ acometimento ocorreu de distal para proximal, o que geralmente ocorre nesses casos com comprometimento em bota (32). Porciúncula et al. (21) constataram em seu estudo grande número de indivíduos que apresentavam ausência do reflexo aquileu na população estudada, principalmente em indivíduos em que já possuíam lesões ulcerativas nos pés.

Quanto à sensibilidade térmica e tátil, observou-se que houve diminuição, já que os estudados apresentam uma vulnerabilidade a lesões e perda da discriminação para quente e frio, chamando a atenção para o inicio da instalação da doença. Em um estudo realizado por Sacco et al. (7), notou-se que antes da intervenção com exercícios físicos, a população estudada apresentava diminuição da discriminação térmica para quente e frio; e, logo após a intervenção, houve melhora significativa nos indivíduos avaliados, com diminuição do risco de eventuais traumas, como queimaduras, proporcionando proteção.

Um dos fatores que predispõem o aparecimento da neuropatia periférica é o valor glicêmico alto, assim como sua flutuação $(7,32)$. Havia na população estudada indivíduos que não faziam o controle adequado da glicemia, o que reflete diretamente nos resultados obtidos pelo Sistema de Pontuação Clínica de Toronto e monofilamento de Semmes-Weinstein. A média da glicemia, de 149,20 $\pm 60,37 \mathrm{mg} / \mathrm{dL}$ e da hemoglobina glicada, de $7,72 \pm 1,4 \%$, indicam que a maioria dos estudados não realizava o controle da glicemia. Isso confirma que a hiperglicemia traz como consequência lesões nos nervos periféricos dos indivíduos diabéticos.

A neuropatia implica na destruição da mielina que reveste as fibras nervosas, tanto sensoriais como motoras resultando em comprometimento na propriocepção e fraqueza. A mielina é essencial para a condução do impulso nervoso por todo o corpo; à medida que um potencial de ação cursa de uma região mielinizada para outra onde a mielina foi danificada, a resistência da membrana diminui. Isso permite o vazamento de corrente, perturbando o fluxo de corrente para o nodo seguinte onde a alta densidade de canais de $\mathrm{NA}^{+}$pode gerar um novo potencial de ação. Com a desmielinização, a condução do potencial de ação se tornará mais lenta e, potencialmente, será bloqueada, resultando em fraqueza motora e anormalidades sensoriais (36).

Rolim et al. (32), em um estudo de revisão, observaram que nos últimos anos, vários estudosmostraram de forma consistente que fatores de risco cardiovasculares têm papel importante no desenvolvimento da neuropatia autonômica, como pressão arterial sistólica (PAS), índice de massa corpórea (IMC), triglicérides e tabagismo. É interessante notar que esses fatores têm pelo menos dois pontos em comum: são potencialmente modificáveis e estão associados à resistência à ação da insulina, sugerindo mais uma vez que a neuropatia pode surgir junto com a síndrome metabólica.

\section{Conclusão}

Nesta pesquisa, a presença de neuropatia, em sua maioria simétrica associada à falta de controle da glicemia com alteração de sensibilidade com presença de sinais clínicos, sintomas, arreflexia e hiporreflexia, era esperada, porém a grande incidência trouxe preocupação e evidenciou a necessidade de avaliação precoce dos pés de todo indivíduo diabético. A associação de outros fatores como obesidade e hipertensão mostra a relevância do acompanhamento da fisioterapia. Ainda evidencia a necessidade de avaliação da sensibilidade para prevenir, atuar na estimulação da regeneração da fibra nervosa, reduzir sintomas e melhorar a circulação sanguínea em MMII.

Uma das limitações do estudo sobre o exercício físico se deve ao fato de não se ter indagado sobre o tipo, duração e tempo em que o exercício vinha sendo realizado. Talvez, munidos desses dados, pudéssemos dissertar mais sobre a correlação da neuropatia com a prática de exercícios. Além disso, há presença de vários outros fatores de risco que podem ter colaborado negativamente com os resultados obtidos a esse respeito.

\section{Agradecimentos}

Os autores agradecem a todos os pacientes que participaram deste estudo e à Associação dos Diabéticos de Bauru (ADB) pela parceria. 


\section{Fontes de financiamento}

O presente estudo foi aprovado no Programa de Iniciação Científica da Universidade do Sagrado Coração (USC) na modalidade Programa de incentivo voluntário a Iniciação Científica (PIVIC - USC).

\section{Referências}

1. Cunha RPF, Brito MMT, Prazeres EMB, Filho NTP. Plasticidade neural e a neuropatia periférica diabética. Fisioter Bras. 2002;3(2):108-15.

2. Costa AA, Almeida JS Neto. Manual de diabetes: educação, alimentação, medicamentos e atividades físicas. 4. ed. São Paulo: Sarvier; 2004.

3. Guyton AC, Hall JE. Tratado de fisiologia médica. 10. ed. Rio de Janeiro: Guanabara Koogan; 2002.

4. Montgomery R, Cowmay TW, Spector AA. Bioquímica: uma abordagem dirigida por casos. 5. ed. São Paulo: Artes Médicas; 1994.

5. Rull JA, Zorrilla E, Jaadzinsky MN, Santiago JV. Diabetes mellitus: complicaciones crônicas. Ciudad de Mexico: McGraw-Hill; 1992.

6. Schmid H, Neumann C, Brugnara L. O Diabetes Melito e a desnervação dos membros inferiores: a visão do diabetólogo. J Vasc Br. 2003;2(1):37-48.

7. Sacco ICN, Sartor CD, Gomes AA, João SMA, Cronfli R. Avaliação das perdas sensório-motoras do pé e tornozelo decorrente da neuropatia diabética. Rev Bras Fisioter. 2007;11(1):27-33.

8. Cosson ICO, Ney-Oliveira F, Adan LF. Avaliação do conhecimento de medidas preventivas do pé diabético em indivíduos de Rio Branco, Acre. Arq Bras Endocrinol Metabol. 2005;49(4):548-56

9. Laurindo MC, Recco DC, Roberti DB, Rodrigues CDS. Conhecimento de pessoas diabéticas acerca dos cuidados com os pés. Arq Ciênc Saúde. 2005;2(2):80-4.

10. Gross JL, Nehme M. Detecção e tratamento das complicações crônicas do Diabetes Melito: consenso da Sociedade Brasileira de Diabetes e Conselho Brasileiro de Oftalmologia. Rev Assoc Méd Bras. 1999;45(3):279-84.

11. Bril V, Perkins BA. Validation of the Toronto clinical scoring system for Diabetic polyneuropathy. Diabetes Care. 2002;25:2048-52.
12. Guedes DP. Composição corporal: princípios, técnicas e aplicações. 2. ed. Londrina: APEFL; 1994.

13. Cronk CE, Roche AF. Race and sex-specific reference data for tríceps and subscapular skinfolds and weight/stature. Am J Clin Nutr. 1982;35(2):347-54.

14. Sociedade Brasileira de Hipertensão, Sociedade Brasileira de Cardiologia, Sociedade Brasileira de Endocrinologia e Metabologia, Sociedade Brasileira de Diabetes, Sociedade Brasileira para estudos da Obesidade. I Diretrizes Brasileiras de Síndrome Metabólica. São Paulo: BG Cultural; 2005.

15. Sociedade Brasileira de Hipertensão, Sociedade Brasileira de Cardiologia, Sociedade Brasileira de Nefrologia. V Diretrizes Brasileiras de Hipertensão. São Paulo: BG Cultural; 2006.

16. Negrato CA. Avaliação clínica do pé diabético. In: Kuhn P, organizador. 0 pé diabético. São Paulo: Atheneu; 2006. p. $17-30$.

17. Sanvito WL. Propedêutica neurológica básica. São Paulo: Atheneu; 1998.

18. Gross JL, Silveiro SP, Camargo JL, Reichelt AJ, Azevedo MJ. Diabetes Melito: diagnóstico, classificação e avaliação do controle glicêmico. Arq Bras Endocrinol Metabol. 2002;46(1):16-26.

19. Barbosa PJB, Lessa I, Almeida Filho N, Magalhães LBNC, Araújo J. Critério de obesidade central em população brasileira: impacto sobre a síndrome metabólica. Arq Bras Cardiol. 2006;87(4):407-14.

20. Tavares EL, Anjos LA. Perfil antropométrico da população idosa brasileira. Resultados da pesquisa nacional sobre saúde e nutrição. Cad Saúde Pública. 1999;15(4):759-68.

21. Porciúncula MVP, Rolim LCP, Garofolo L, Ferreira SBG. Análise de fatores associados à ulceração de extremidades em indivíduos diabéticos com neuropatia periférica. Arq Bras Endocrinol Metabol. 2007;51(7):1134-42.

22. Grossi SAA. Prevenção de úlceras nos membros inferiores em indivíduos com Diabetes Mellitus. Rev Esc Enferm USP. 1998;32(4):377-85.

23. Solórzano JB. El impacto del fumado y sus efectos sobre la enfermedad de la diabetes mellitus. Rev Med Costa Rica y Centroamerica. 2006;63(577):163-7. 
24. Gamba MA, Oliveira O, Fraige F Filho, Martinez C, Kajita MY. A magnitude das alterações cutâneas, neurológicas, vasculares de extremidades inferiores de pessoas com diagnóstico de diabetes mellitus. Campanha de detecção e educação da ANAD. Diabetes Clínica. 2001;5(6):414-8.

25. Triana MH. Alteraciones Metabolicas en el alcoholismo. Rev Cubana Aliment Nutr. 1996;10(1):35-42.

26. Gomes AA, Sartor CD, João SMA, Sacco ICN, Bernik MMS. Efeitos da intervenção fisioterapêutica nas respostas sensoriais e funcionais de diabéticos neuropatas. Fisioter pesqui. 2007;14(1):14-21.

27. Luciano E. Atividade física e metabolismo lipídico em ratos diabéticos experimentais. Rev bras ativ fís saúde. 1996;1(4):19-26.

28. Klack K, Trecco SMLSS, Moura NF. Reabilitação nutricional para diabéticos. Diabetes Clínica. 2007;11(3):266-75.

29. Geloneze B, Tambascia MA. Avaliação laboratorial e diagnóstico da resistência insulínica. Arq Bras Endocrinol Metabol. 2006;50(2):208-15.

30. Picon XP, Zanatta CM, Gerchman F, Zelmanovitz T, Gross JL, Canani LH. Análise dos critérios de definição da síndrome metabólica em indivíduos com diabetes melito tipo 2. Arq Bras Endocrinol Metabol. 2006;50(2):264-70.

31. Ochoa-Vigo K, Pace AM. Pé diabético: estratégias para prevenção. Acta Paul Enferm. 2005;18(1):100-9.
32. Rolim LCSP, Sá JR, Chacra AR, Dib SA. Neuropatia autonômica cardiovascular diabética: fatores de risco, impacto clínico e diagnóstico precoce. Arq Bras Cardiol. 2008;90(4):24-32.

33. Schimid H. Impacto cardiovascular da neuropatia autonômica. Arq Bras Endocrinol Metabol. 2007;51(2):232-43.

34. Lira JRS, Castro AA, Pitta GBB, Figueiredo LFP, Lage VMM, Miranda F Júnior. Prevalência de polineuropatia sensitivo-motora nos pés no momento do diagnóstico do diabetes melito. J Vasc Br. 2005;4(1):22-6.

35. Boyd BS, Wanek L, Gray AT, Topp KS. Mechanosensitivity during lower extremity neurodynamic testing is diminished in individuals with type 2 Diabetes Mellitus and peripheral neuropathy: a cross sectional study. BMC Neurology. 2010 [acesso 12 ago 2013];10(75). Disponível em: http://www.biomedcentral.com/1471-2377/10/75.

36. Lundy-Ekman L. Neurociência: fundamentos para a reabilitação. Rio de Janeiro: Guanabara Koogan; 2000.

Recebido: 15/07/2012 Received: 07/15/2012

Aprovado: 13/02/2013 Approved: 02/13/2013 